\title{
Mushroom-bodies regulate habit formation in Drosophila
}

\author{
Björn Brembs \\ Freie Universität Berlin, Inst. Biol. - Neurobiologie \\ bjoern@brembs.net
}

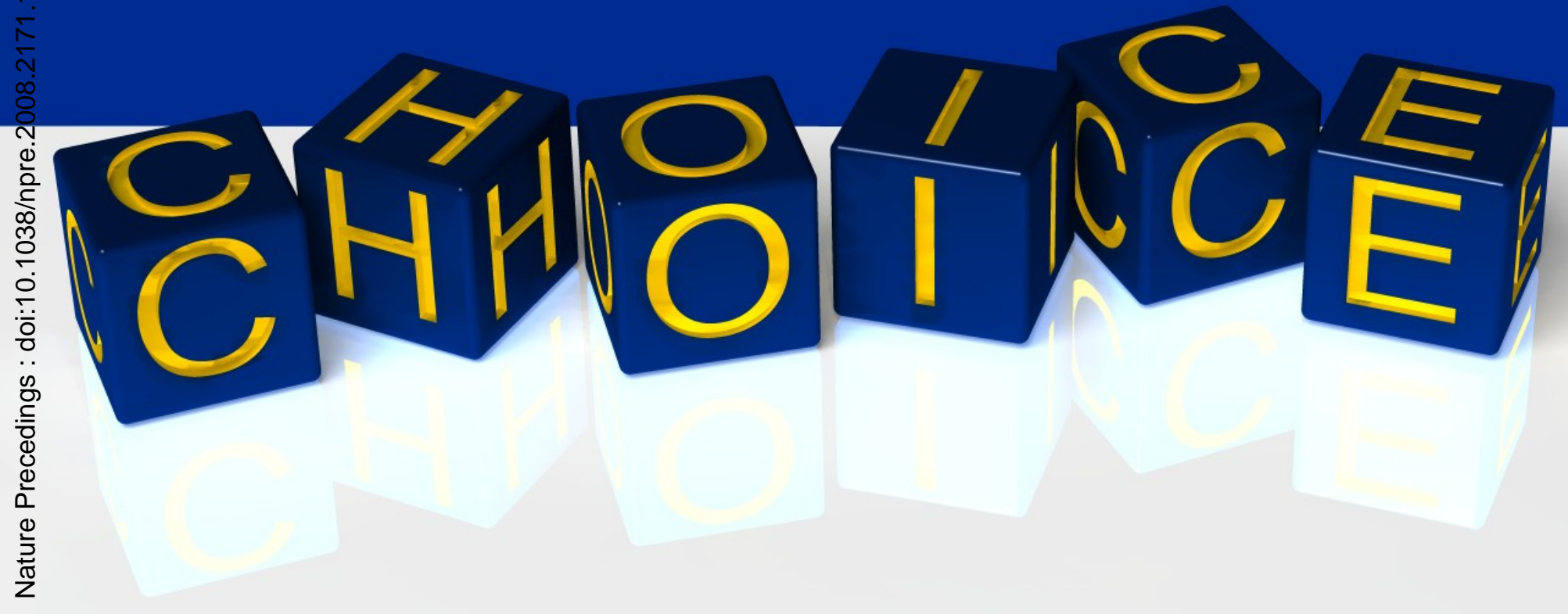

FENS Forum 2008 in Geneva, Switzerland

S28: The neurobiology of choice and decision-making . 


\section{Decisions, Decisions}

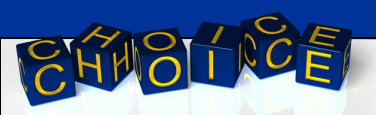
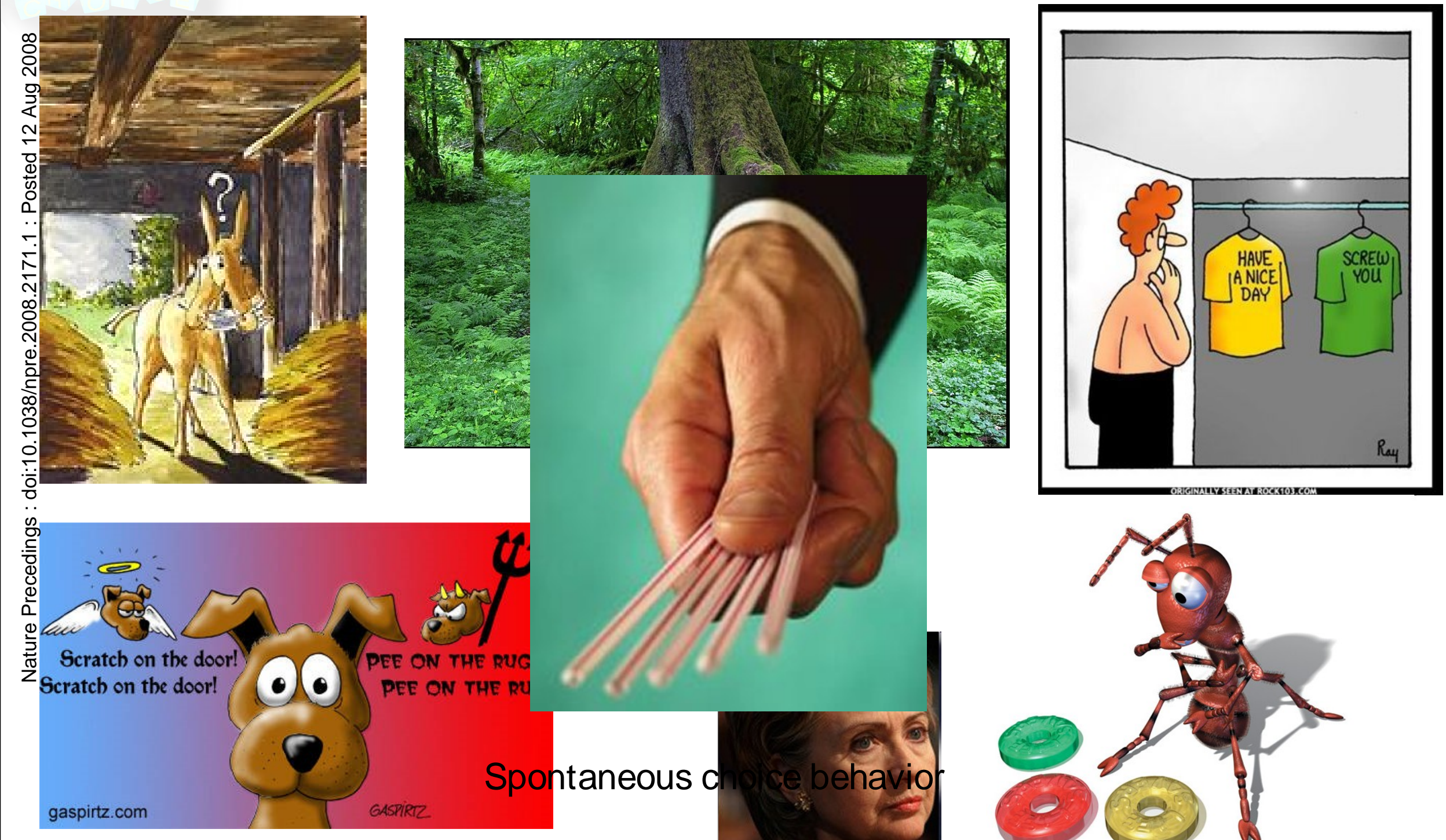

4

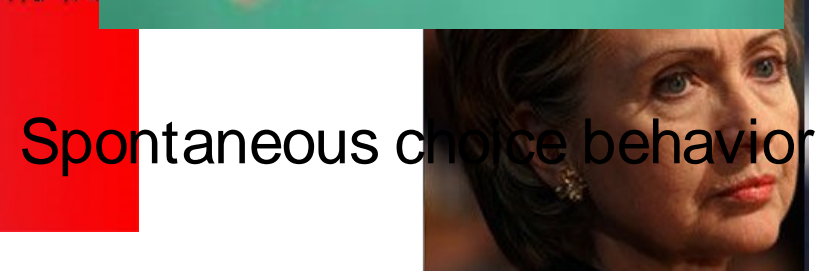




\section{Drosophila at the Torque Meter}

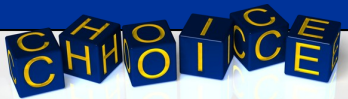

Spontaneous choice in a constant environment

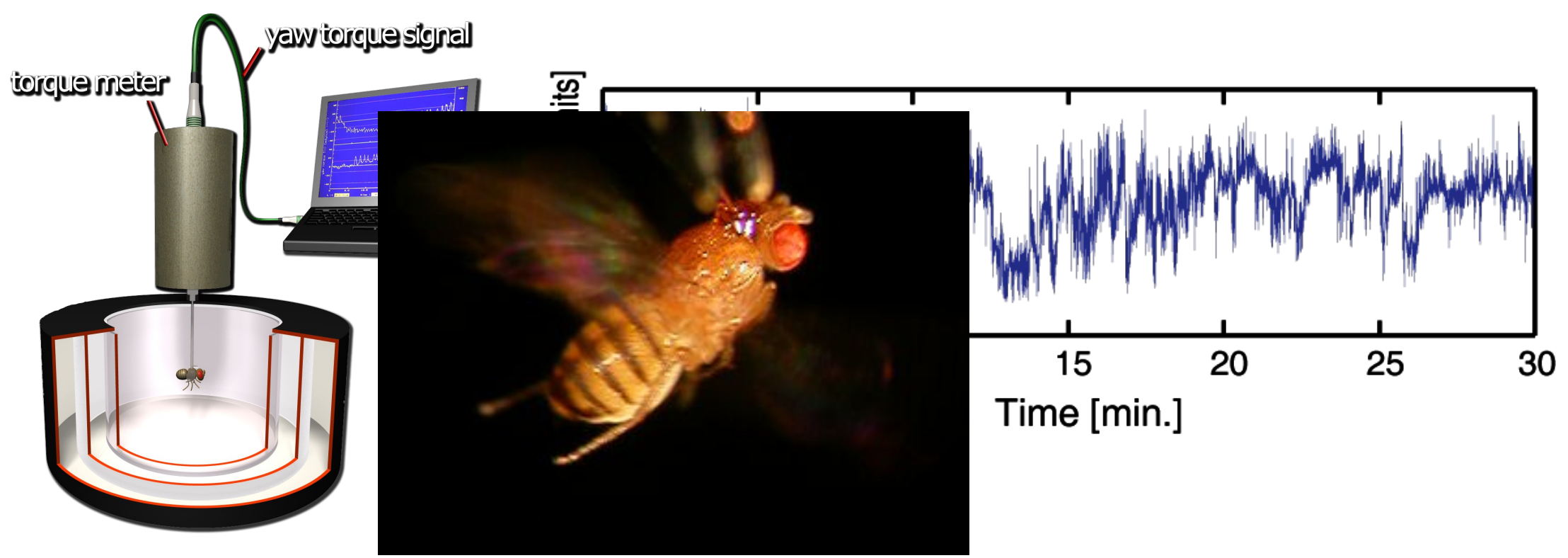

How flies use re-afferent feedback to modulate this spontaneous choice behavior 


\section{Feedback Stimuli}

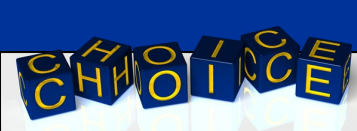

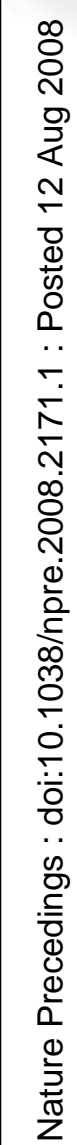
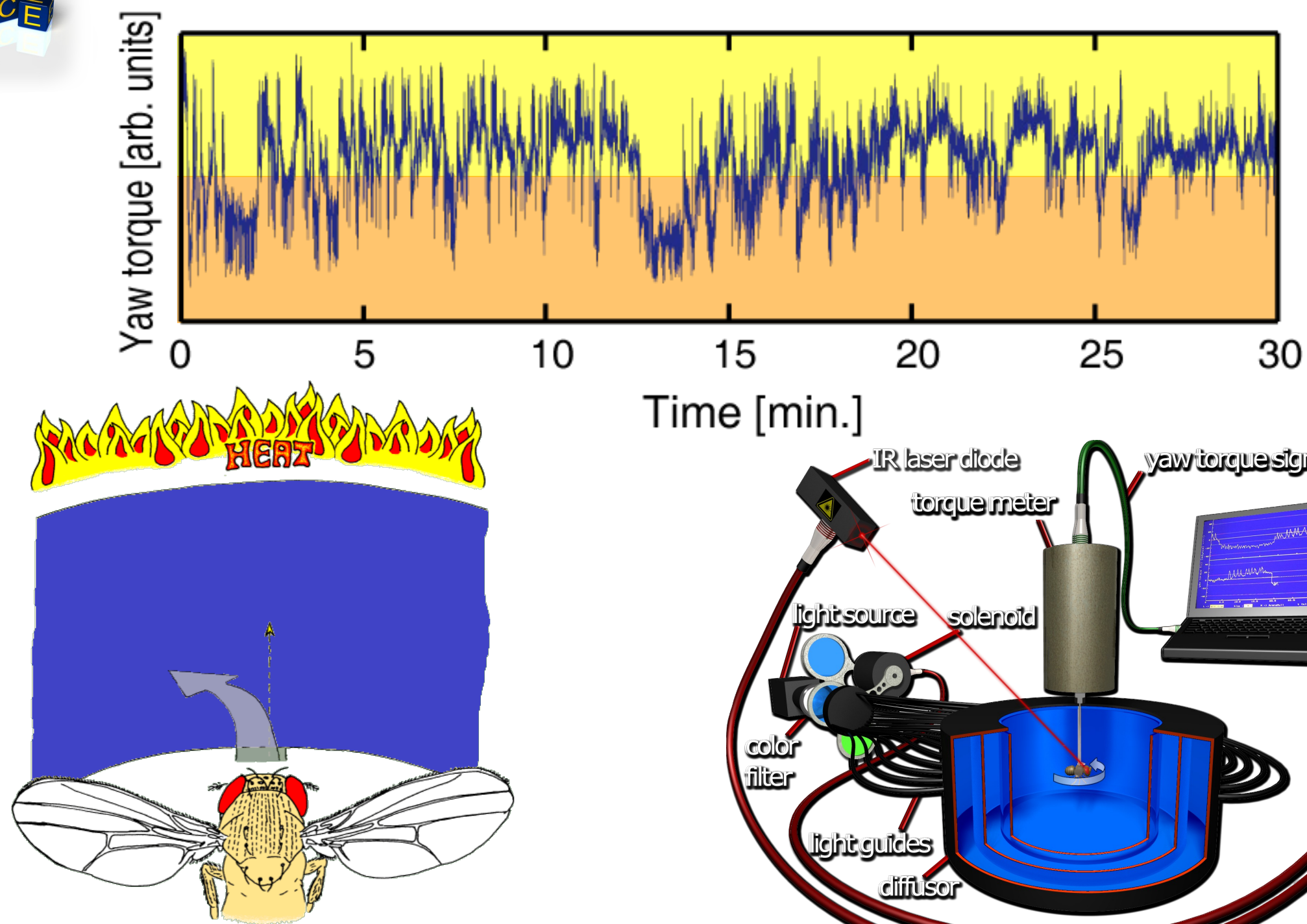

Time [min.]

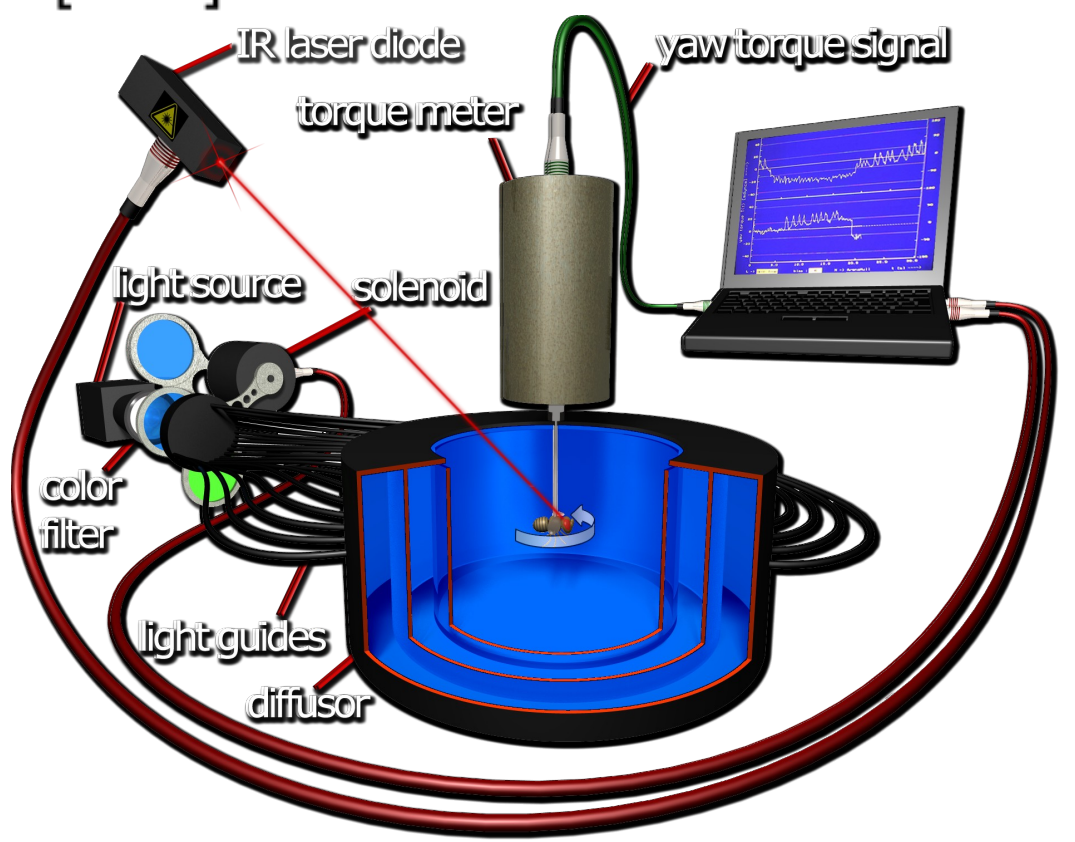

Fly drawing courtesy of Reinhard Wolf 


\section{Isolating the Components}

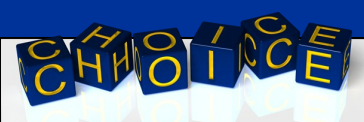
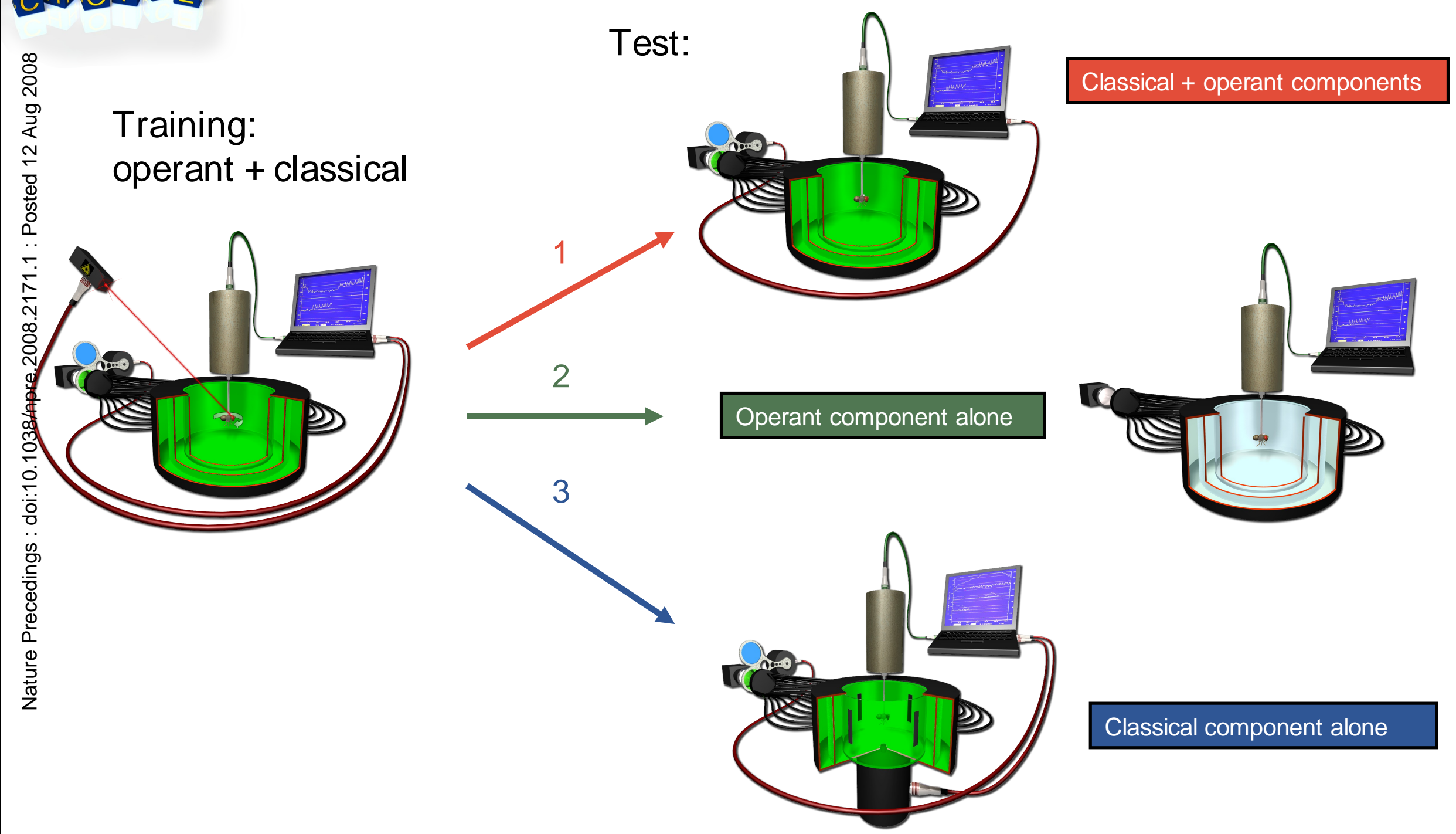

Brembs \& Heisenberg (2000) 


\section{Mushroom-Bodies central}

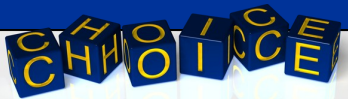

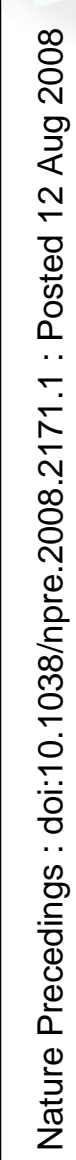

...for keeping classical memories flexible for generalization
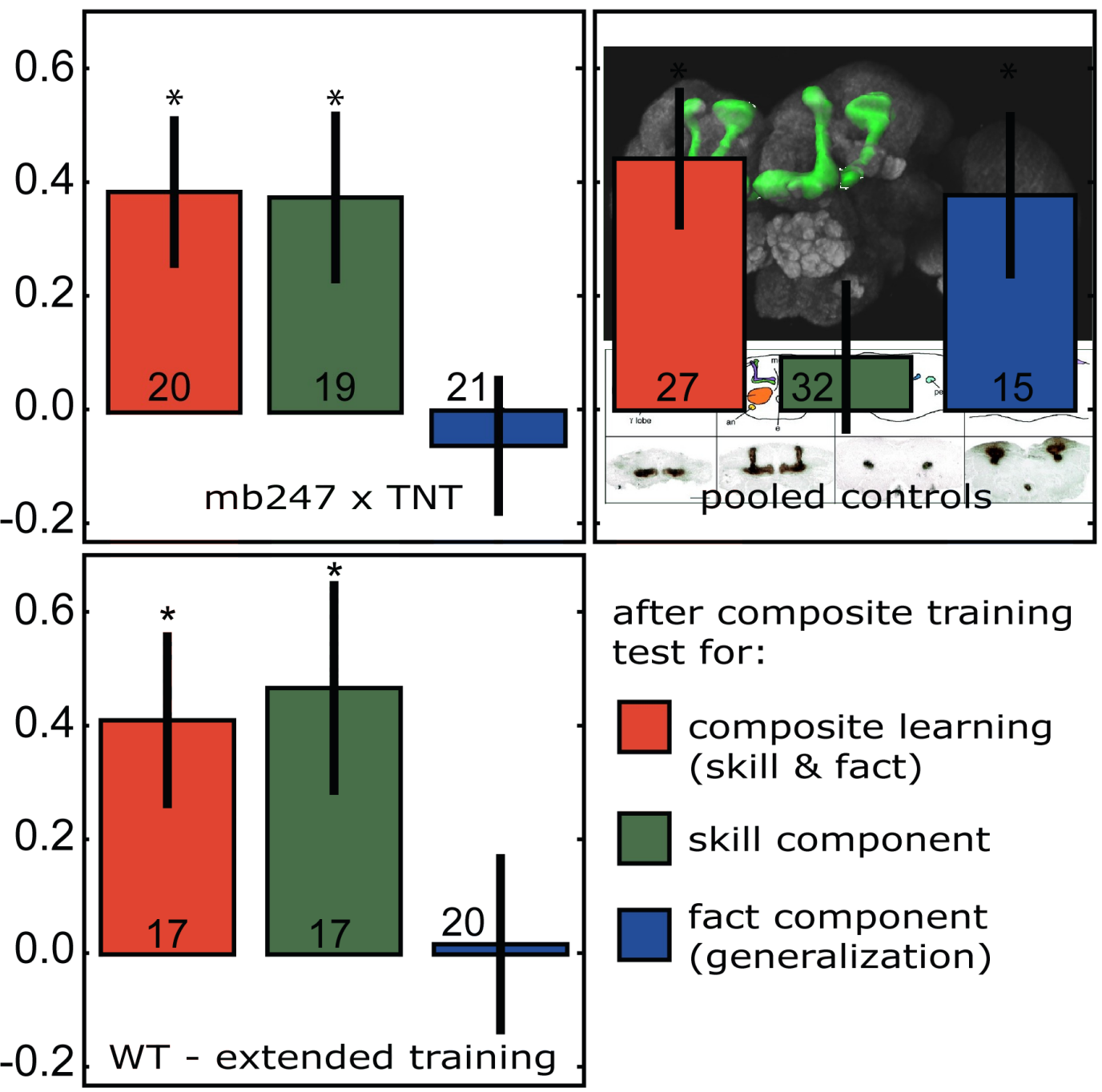

- Mushroom-bodies mediate inhibition of operant learning

- Mushroom-bodies enable flexible generalization

- Mushroom-bodies delay acquisition of stereotyped, habitual responses

after composite training test for:

composite learning (skill \& fact)

$\square$ skill component

fact component (generalization)

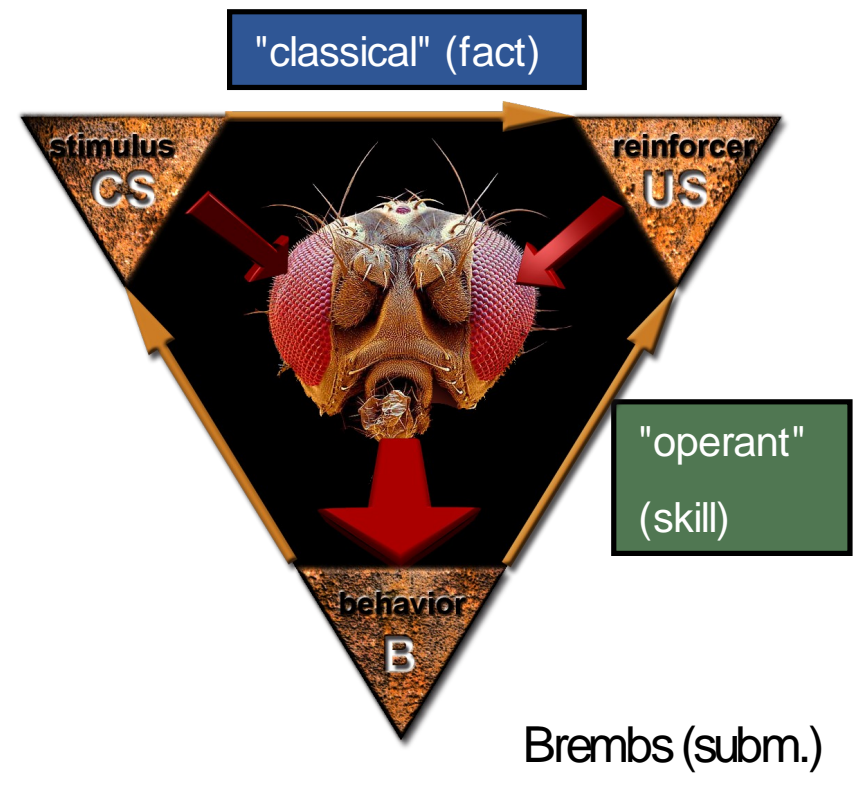




\section{Complex, Hierarchical Interactions}

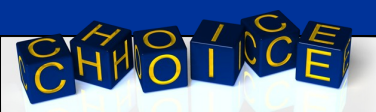

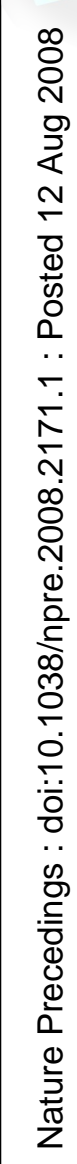

between multiple memory systems instead of a simple dichotomy

\section{Composite learning}

ACdependenน

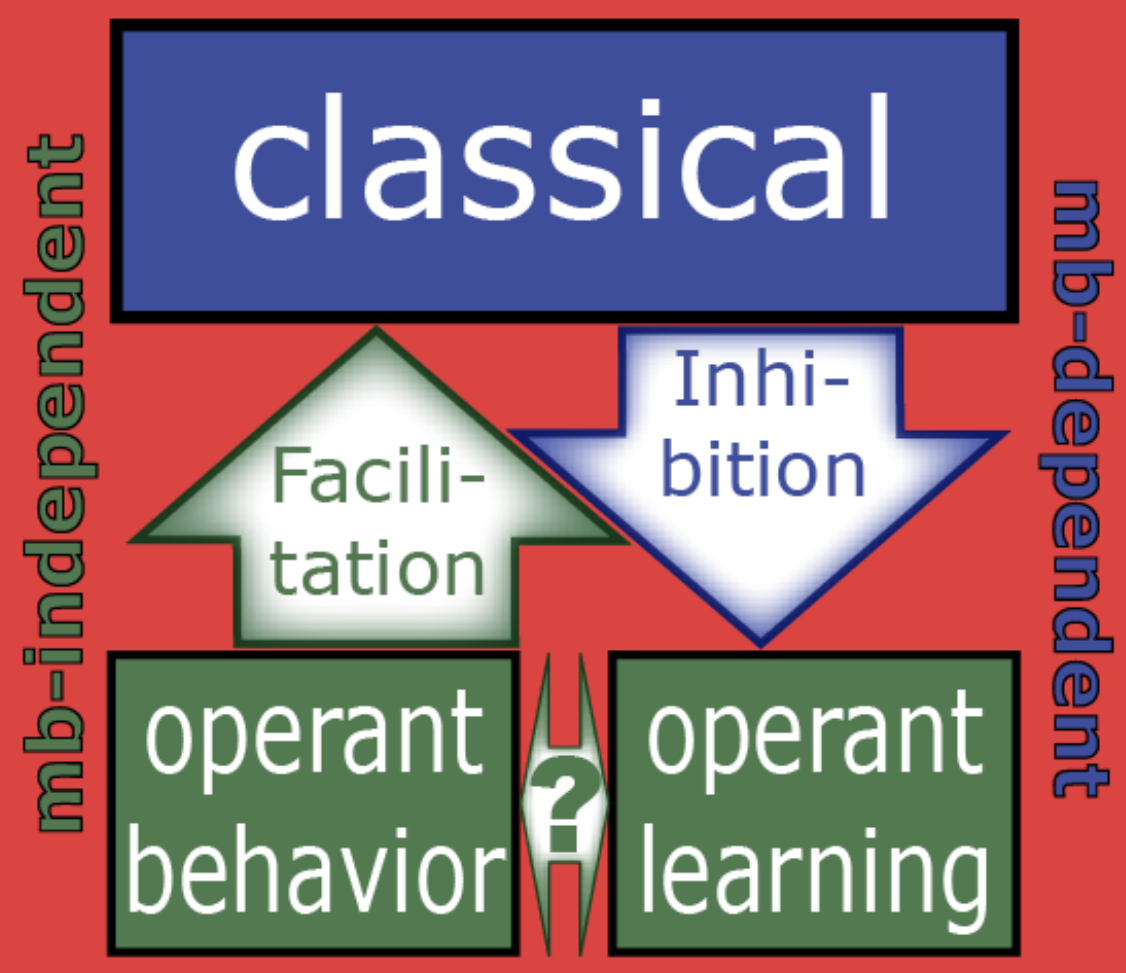

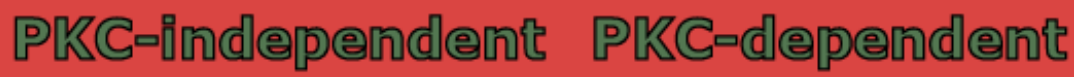

